\title{
When did you stop speaking to yourself? Adolescent development of world knowledge-based audience design
}

\author{
Caroline Arvidsson ${ }^{1}$, David Pagmar ${ }^{1}$, and Julia Uddén ${ }^{1,2}$ \\ ${ }^{1}$ Dept. of Linguistics, Stockholm University \\ ${ }^{2}$ Dept. of Psychology, Stockholm University
}

\begin{abstract}
The ability to adapt utterances to the world knowledge of one's addressee is undeniably ubiquitous in human social cognition, but its development and association with other cognitive mechanisms during adolescence have not been studied. In an online production task, we measured the ability of children entering adolescence (ages 11-12) and adolescents (ages 15-16) to tailor referential expressions in accordance with the inferred world knowledge of their addressee - an ability we refer to as world knowledge-based audience design. A post-test survey showed that relevant theory of mind information was accessed upon prompting in both age groups, but the younger age group did not consistently utilize this information during online production, resulting in a significantly improved world knowledge-based audience design across age groups. We also investigated the reliance of world knowledge-based audience design on cognitive control functions. Cognitive control (as reflected by performance on the Wisconsin card sorting task) increased significantly with age, but did not explain the age-related increase in audience design performance. We thus provide evidence for an adolescent development of world knowledge-based audience design over and above development of theory of mind and cognitive control.
\end{abstract}

Keywords: Audience design, pragmatic development, referential production, adolescence, theory of mind, cognitive control functions.

\section{INTRODUCTION}

When describing the general conversational conduct of young children, Piaget stated that children under the age of 7 virtually "speak to themselves" [1]. This can be seen as an early observation that children do not engage in what is frequently termed audience design (AD) [2] to the same extent as adults [3,4]. AD entails adjusting utterances to suit the needs of interlocutors. This listener-catering behavior operates on all levels of language, but is particularly salient in referential production, in which speakers often need to take into account the world knowledge of their interlocutor. For example, consider a situation in which a pragmatically skilled speaker wishes to refer to her favorite computer game. If "computer games" is not assumed to be an area of knowledge for the addressee (e.g. her 90 year-old grandmother), the speaker will be inclined to produce a more informative noun phrase (e.g. "The game that mom bought me for my birthday") instead of a proper name (e.g. "Minecraft") to maximize the possibility of overlap between her and her interlocutors' mental representation as to what is being denoted. From a Gricean perspective, engaging in world knowledge-based $\mathrm{AD}$ means adhering to the Gricean maxim of quantity [5], by making one's conversational contribution adequately informative for a particular addressee. But if children do not engage in world knowledge-based $\mathrm{AD}$ to the same extent as adults $[3,6]$, when do individuals become adult-level proficient communicators in this respect? No previous study has investigated the developmental trajectory of this ability in late childhood and adolescence. We aim to address this gap in the literature and further the understanding of how AD develops throughout the life span.

Our approach is built on the processing perspective prominent in Clark [7]. An important input to the process of tailoring utterances to the assumed knowledge state of one's interlocutor (i.e., the AD process) is the discernment between what is common ground (knowledge shared by both interlocutors) [2], and what is privileged ground (knowledge exclusively available to the speaker) [8]. Viewing common ground as the intersection of the speaker's and the addressee's area of knowledge with respect to a given 
referent, the speaker's process of modelling common ground could be construed as including a step where aspects of theory of mind (ToM) are used. ToM is known as a collection of concepts that inter alia allow individuals to perceive others as mental beings driven by their own knowledge state [9, 10]. From the processing perspective, possession of ToM information can be distinguished from online ToM usage. In what follows, ToM information denotes the speaker's assumption regarding the addressee's knowledge state. Online ToM usage, on the other hand, stands for the process in which speakers utilize ToM information in order to adapt their utterances accordingly. In the current study, we investigate the development of world knwoledge-based AD in late childhood (11-12) and adolescence (15-16) by utilization of a task design that distinguishes between these aspects. We present a novel AD task and scoring method that requires speakers to utilize ToM information on the assumed world knowledge of interlocutors during online referential production.

\subsection{Development of ToM and and ToM usage during online communication}

As ToM is an ability with many facets, we will focus on aspects needed for consistent production of adequately informative utterances. One plausible prerequisite is the insight that others' knowledge states can differ from one's own. Children exhibit this ability from a young age [11]. For example, in classical false-belief tasks, 3- to 4-year-olds are able to keep track of and reproduce specific information that a playpartner has not seen due to being absent at the time when the information was provided [12]. Interestingly, 4- to 6-year-olds do not utilize ToM information regarding the visual perspective of interlocutors during online production to the same extent as adults $[4,13]$. This suggests that while relevant ToM information as such may be present and accessible for children, it is not always utilized during online production. Such a pattern was observed in a study on 10-year-olds [14], showing that while children are able to model common ground with respect to what has been mentioned in the previous linguistic context, they do not consistently deploy the modelled common ground information during production. Possibly, the ability to utilize ToM information online in an adult-like manner develops much later. In a comprehension study [15], it was found that the ability to use ToM information regarding the speaker's perspective in order to correctly choose a target referent develops throughout adolescence (ages 11-18). This suggests that ToM usage during online communication continues to develop well beyond the pre-school years. In addition, the ability of engaging in $\mathrm{AD}$ may depend on which kind of ToM information is used online (for example, visual perspective or world knowledge).

\subsection{Development of AD during online referential production}

Because of its universality and simplicity, referential production has gained substantial attention within AD research $[16,17]$. Traditional referential communication tasks aim to measure the ability to adapt utterances to the knowledge state of addressees, typically by modifying interlocutors' visual access to referents. An example of such a task is the well-established "director task" (DT) [18]. Performance on the DT (specifically in the "common ground condition") is typically assessed by counting utterances in which participants use disambiguating adjectives to guide an addressee in choosing one specific object among multiple competing objects displayed [4]. Children are less effective than adults at making their referential expressions as informative as required in these types of tasks. For example, in only $39 \%$ of these trials, 4- to 5-year-olds provided disambiguating adjectives [13], while 5-6-year-old children used disambiguating adjectives in $75 \%$ of trials [4], and adults provided disambiguating adjectives in $100 \%$ of trials [4]. This suggests that AD in referential production develops successively during childhood, and that children reaching school-age have not yet developed into adult-level proficient referential communicators.

Less is known regarding the development of $\mathrm{AD}$ in referential production in adolescence. However, in one DT study [19], 11-16-year-old participants provided disambiguating adjectives when necessary from the addressee's perspective nearly as often as adults, but failed to omit size-adjectives when redundant. Albeit these findings may reflect a true late and successive development of the ability to take information concerning the listener's visual perspective into account, AD-development in adolescence was not the focus of the study, i.e., variation within 11-16-year-olds was not investigated. In addition, utterance adaptation to the visual perspective of interlocutors is arguably significantly less prevalent in real-life conversation than adaptation to the assumed world knowledge of interlocutors. We wanted to improve the state of this literature along these lines, as the DT task has been criticized for not necessarily tapping online ToM usage, but other domain-general cognitive functions such as selective visual attention [20]. As we recently conducted a world knowledge-based AD task (similar to the task in the current study) with 
children, which showed that 7-year-olds fail to adapt their utterances to the addressee's world knowledge in more than half of trials [3], we expected to see an increase in this ability throughout adolescence.

In the current study, we investigated the development of $\mathrm{AD}$ in referential production, using an innovative task design $[3,6]$, that required speakers to use ToM online by considering the world knowledge of interlocutors, rather than their visual access to referents. We compared performance on this task in two age groups: children entering adolescence (11-12) and mid adolescents (15-16). While views regarding at which age adolescence is reached vary across cultures, most researchers agree that its commencement coincides with the onset of puberty [21]. We hypothesized that the ability to utilize ToM information during online production and thus the ability to engage in world knowledge-based AD develops in these age-ranges. This is a time when an individual's social world becomes increasingly peer-oriented and complex [21], and the prediction is consistent with the developmental literature on ToM usage (see section 1.1). Additional support for this hypothesis was the observation that adults' perspective-taking during referential production is subserved by medial prefrontal and temporo-parietal regions [22] - regions that undergo structural and functional changes during adolescence [21]. An increase in world knowledge-based $\mathrm{AD}$ in the investigated age-ranges could reflect a development of ToM as such, or of ToM usage during online production, and we crucially addressed this issue experimentally (see section 2.3.2). As ToM usage during online communication has been hypothesized to rely on more domain-general cognitive control functions [23], we now turn to this possibility.

\subsection{The role of cognitive control functions in online communicative ToM usage}

Recall that young children show awareness of others' perspectives [12], but fail to consistently use this information during conversation $[3,4,13]$. Building on this literature, it was hypothesized that usage of ToM during online communication relies on the development of domain-general cognitive control functions ${ }^{1}$ (CCF) [13]. Again, taking the processing perspective, the term CCF denotes a set of domain-general, higher order processes that facilitate flexible and goal-oriented behavior [24]. In online production, it has been proposed that $\mathrm{CCF}$ facilitates common ground modelling during online production by (1) suppressing an initial "egocentric" perspective, (2) shifting to a listener-friendly perspective, and (3) maintaining and updating relevant contextual, social and linguistic information relevant to one's conversational contribution [23]. Roughly, these suggestions match the three well-studied components of CCF: inhibition, cognitive flexibility and working memory [25-27]. In an extensive review [28], it was concluded that while $\mathrm{CCF}$ usually correlate with global measures of pragmatic ability, the relation between $\mathrm{CCF}$ and specific pragmatic abilities remains unsettled.

Developmentally, no link between CCF (working memory, inhibition and cognitive flexibility) and the ability to provide sufficiently detailed referential expressions was observed when younger children participated in a visual perspective-taking task (years: 4-5) [13], whereas a correlation between CCF subfunctions (cognitive flexibility and working memory) and children's (ages 4-6 years) ability to successfully produce sufficiently detailed and repair insufficient referential expressions was observed [29]. On the adult side, the literature is also mixed, but a recent large-scale study found no correlation between adults' AD performance and their working memory capacity (see [6] and references therein).

In order to investigate whether a potential increase of ToM usage during production across age-groups reflects a development of processes special to the communicative situation rather than general cognitive control functions, we measured CCF using the Wisconsin card sorting task (WCST) (see further section 2.3.3.). Due to mixed evidence, we hypothesized that although some of the potentially observed ADdevelopment might be explained by adolescents' $\mathrm{CCF}, \mathrm{AD}$ development would also manifest over and above CCF development.

\subsection{Summary of the approach}

In order to answer the questions regarding the development of world knowledge-based $\mathrm{AD}$ in adolescence, we conducted a novel version of the DT task, requiring speakers to utilize ToM information regarding the world knowledge, rather than visual perspective, of addressees. We expected a significant increase in AD performance as reflected by an increased ability to use ToM during online referential production, even when controlling for a probable increase of ToM information and CCF across age groups.

${ }^{1}$ Cognitive control functions $(\mathrm{CCF})$ is here used as a term interchangeable with executive functions. 


\section{METHODS}

\subsection{Participants}

The sample consisted of 58 adolescents from two age groups (11-12 years: $N=29,17$ girls; $15-16$ years: $N=29,17$ girls), recruited from two schools in Värmdö municipality, Stockholm County. The two participating schools (one middle school and one high school) by which information about the study was disseminated, were chosen due to their close proximity; many of the pupils attending the participating high school had previously attended the participating middle school.

The study was approved by the Swedish Ethical Review Authority (Dnr 2020-07083). Informed consent was obtained from all participants and the legal guardian(s) of participants in the younger age group. Participants received small tokens of appreciation (value $\leq 50 \mathrm{SEK}$ ) upon participation. Participants were (1) fluent speakers of Swedish and (2) had not been diagnosed with any language impairment, as ascertained by a parental-report (younger age group) or self-report (older age group) questionnaire. Three participants were excluded prior to analysis due to reports of having been diagnosed with ASD, ADHD and/or DLD. One participant was excluded after having more than half of trials deleted during the analysis of a post-test survey (section 2.3.2) that controlled for knowledge of referents and assumption about listeners' knowledge of stimuli (see further section 2.4.1).

\subsection{Materials and procedure}

During one full half hour session, the participant was presented with two tasks respectively designed to measure (1) audience design (AD) in referential production and (2) cognitive control functions (CCF).

\subsection{Tasks}

\subsubsection{Test of audience design (AD)}

The referential production task was modified from two prior studies $[3,6]$. Its aim was to measure adolescents' ability to adapt their referential expressions to the inferred world knowledge of their interlocutor. In each trial, a picture of the addressee (either a child or an elderly woman) was presented in the upper corner of a laptop screen. At the bottom of the screen, one target referent (e.g. a video game character, a musician, or other individual/object) and three competitor referents were displayed. In both of the task's conditions, the participant knew the name of the target referent but was expected to assume that the addressee either (1) did not know the name of the target (unknown test condition) or (2) did know the name of the target (known control condition). An example of four trials from the task's two conditions is given in Figure 1. Participants' knowledge of each target referent and their assumptions regarding the addressee's knowledge of each target referent were controlled for through a post-test survey (see section 2.3.2). Successful referential production entailed describing the target referent, i.e., providing at least one visual feature of the target referent by for example uttering a modified noun phrase (see Table 1), rather than simply naming the referent, when the participant assumed that the listener did not know the name of the target referent.

During the practice trial, the participant was instructed to produce an utterance that would guide their addressee to choose the target picture. Because pilot runs of the experiment showed that participants that were initially presented with an unknown condition trial described referents throughout the entire experiment (irrespective of trial condition), the practice trial was always a known condition trial (in which referring by describing was redundant) in order to avoid that participants had misunderstood the task by thinking that they were supposed to consistently describe target referents. Thus, if the participant referred to the target in the practice trial as 'The tree with a star', the experimenter always said 'Sure, but you could also just say Christmas tree, right?'.

To control for processing efforts of maintaining disambiguating features of competing referents in memory, the competitor stimuli were chosen so that a noun phrase containing no more than one modifier (e.g. an adjectival or a prepositional phrase) was enough to comprise an unambiguous target description (see Table 1). Furthermore, the selected stimuli were adapted so that target referents would be known to adolescents, and also could be assumed by adolescents to be either unknown or known to specific addressees, depending on the listener's age and gender. This was verified through an online questionnaire with six respondents (age 11) that were not part of the experiment.

Building on results from 7-year-olds [3], we assumed that participants would name target referents in the known trials and that a relatively small number of trials would suffice to record that behavior. We however expected that pragmatic development (taking place between age 7 through the investigated 


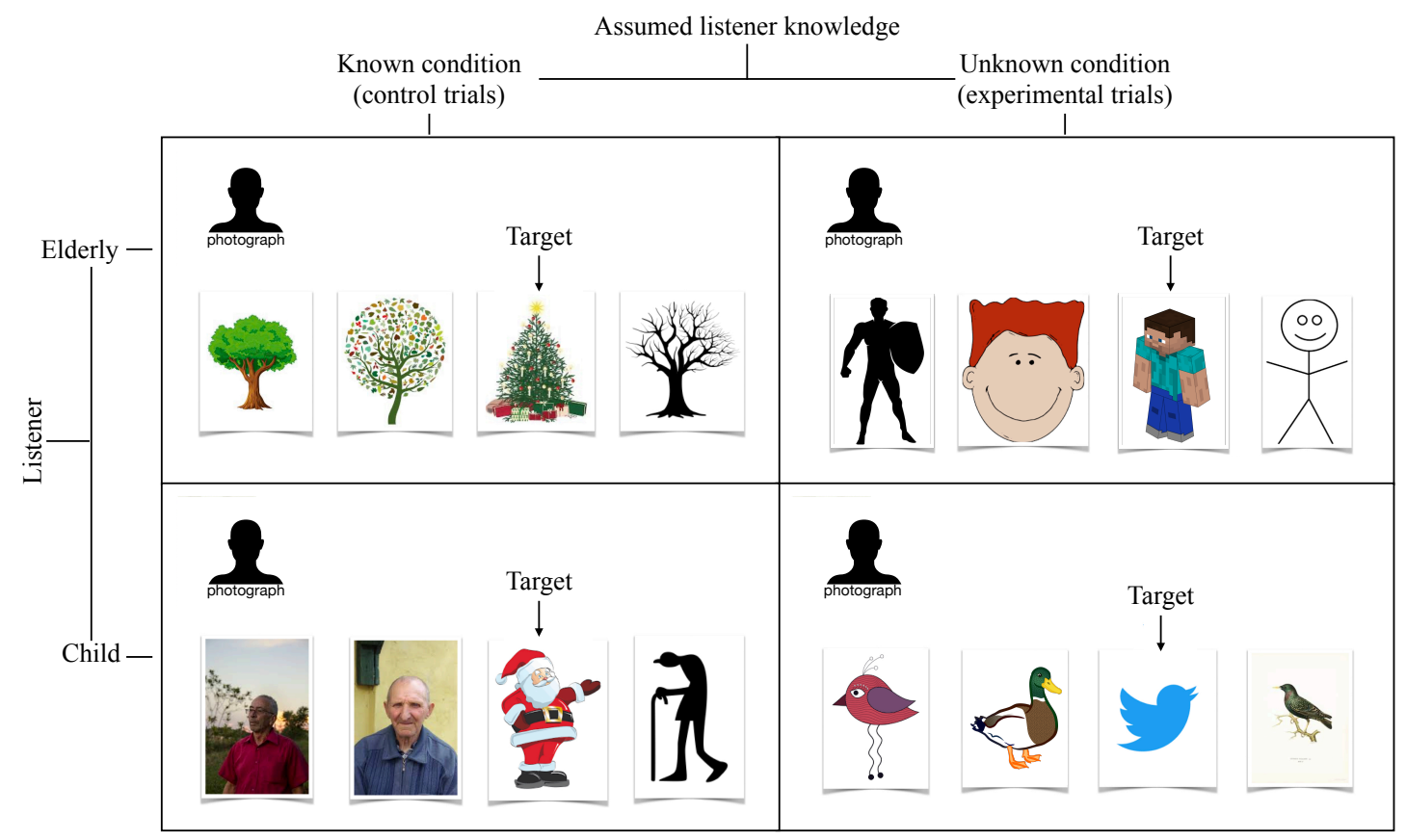

Figure 1. Example of four trials showing the AD task's two conditions. In the upper corner was a photograph of the listener. Target referents in known condition: Christmas tree, Santa Claus. Targets in unknown condition: Minecraft character, the Twitter logo. Images are in public domain (CC0 1.0).

Table 1. Translated examples (from Swedish) of descriptions of target referents. To provide referential expressions that successfully distinguished targets from competitors, a noun phrase with a maximum of one modifier (an adjectival or prepositional phrase) was necessary.

\begin{tabular}{lll}
\hline Target & Competitors & Example of target description \\
\hline Christmas tree & Trees & 'The tree with a star' \\
Santa Claus & Elderly males without beards & 'The bearded man' \\
Minecraft character ${ }^{2}$ & Cartoon figures (none in t-shirt) & 'The man in blue pants' \\
Twitter logo & Pink, white or greenish birds & 'The blue bird' \\
\hline
\end{tabular}

age groups in the present study) would be observed as variability in the unknown trials, i.e., that not all participants would show the more pragmatically developed behavior of describing referents based on assumed lack of listener knowledge. For this reason, we doubled the number of trials in the unknown condition. Altogether, the task consisted of 36 trials (24 in the unknown condition, 12 in the known condition). Four randomized versions of the experiment were presented in an evenly balanced fashion across participants. The test was implemented using PsychoPy [30].

Scoring the AD task, we wanted to (1) get an overall estimate of how inclined the participant was to flexibly switch referring strategy (describing/naming) in accordance with their assumptions about the addressee's knowledge state, and (2) account for the possibility that the participant solely used describing as an overall strategy. Thus, individual performance on the AD task was computed by subtracting the proportion of sufficient (i.e., describing) referential expressions in the unknown (experimental) condition with the proportion of redundant (i.e., describing) referential expressions in the known (control) condition. This resulted in an AD score somewhere between -1.0 (participants who only described when describing was redundant) and 1.0 (participants who only described when describing was necessary).

\subsubsection{AD post-test survey: Assessing ToM information}

We wanted to investigate whether participants' assumptions regarding the knowledge state of their addressee matched a "common sense" view of what the addressee should and should not know due to 
factors such as age and gender (i.e. whether participants' ToM information matched expected ToM). Participants were presented with pictures of each target referent from the AD task. They were furthermore asked if they believed that the addressee (the child or the elderly woman) knew the name of the referent. Trials in which participants ToM matched expected ToM were labelled matching ToM trials. Trials in which participants' assumptions did not match the "common sense" view were labelled non-matching ToM trials. Based on this data, we computed a knowledge attribution score (KAS). KAS was defined as the number of matching ToM trials, divided by the total number of trials. This resulted in a value between 0.0 and 1.0. We included the KAS score in the model to explicitly test for the possibility that development of AD may be due to ToM development.

\subsubsection{Test of cognitive control functions (CCF)}

To assess adolescents' CCF, a computerized version of the Wisconsin card sorting task (WCST) was administered. The WCST is one of the most frequently used neuropsychological measures of CCF [31-34]. Participants matched cards according to a hidden matching rule (color, number or shape) that changed implicitly after 10 consecutive trials. The task required participants to figure out the rule by trial and error with feedback responses after each matching. Two WCST scores were utilized: proportion perseverative errors (PE) and proportion non-perseverative errors (NE). A PE was defined as a failure to switch matching rule after a set shift. An NE was defined as any error that was not perseverative. Both PE and NE were predicted by measures of cognitive flexibility and working memory capacity in adolescence [35, 36]. Conceptually, a PE represents inability to flexibly shift matching-strategy when required and an NE represents inability to maintain matching-strategy when required [37]. The computerized implementation of WCST used in the current investigation consisted of 72 trials (including 12 practice trials) and was available at PsyToolkit's $[38,39]$ experiment library ${ }^{3}$.

\subsection{Analysis}

\subsubsection{Data processing}

As planned, we removed data from (1) trials in which the participant did not know the name of the target referent (6\% of trials) and (2) non-matching ToM trials (5\% of trials). One participant was excluded from the analysis, since as many as 17 trials (12 non-matching ToM trials, indicating low ToM) were removed from this particular participant. The remaining participants $(N=58)$ had a minimum of 13 trials $(\mathrm{M}=$ $20.44)$ in the unknown condition and a minimum of 10 trials $(M=11.68)$ in the known condition (starting number of trials: 24 unknown condition, 12 known condition).

A Shapiro-Wilks normality test showed that AD data was positively skewed (data were normally distributed for the younger age group, whereas $66 \%$ of the older adolescents had a score of 0.7 or higher, with the maximum and minimum possible scores being 1.0 and -1.0). Thus, data were transformed using a rank-based inverse normal (RIN) transformation, which, when compared to a wide range of transformation methods, have been shown to be most beneficial with respect to statistical power on moderate sample sizes $(N \geq 20)$ [40]. All data and scripts are available at [41].

\subsubsection{Statistical analyses}

One frequentist univariate generalized linear model (GLM) (Model 1a) and one corresponding Bayesian GLM (Model 1b) were used to test AD as a function of (1) age, (2) matching ToM information and (3) CCF. In Model 1a and Model 1b, AD score was dependent variable and the independent variable testing for (1) was AGE GROUP (11-12 vs. 15-16), while KAS tested for (2) and PE and NE tested for (3).

Three ANOVAs for independent samples were conducted to investigate whether ToM information and CCF depended on age group. In all three ANOVAs, AGE GROUP was an independent variable. In the first ANOVA, KAS was dependent variable (Model 2), in the second PE was dependent variable (Model 3 ) and in the third NE was dependent variable (Model 4).

All analyses were conducted with the statistical software R [42]. Frequentist models were fit using the built-in functions $\mathrm{glm}$ and aov with an alpha level of $\alpha=0.05$. The Bayesian model was fit using package rstanarm (function stan_glm) [43]. Package bayestestR (function bayesfactor_parameters) [44] was used to calculate Bayes factor ${ }_{10}\left(\mathrm{BF}_{10}\right)$ with default priors. $\mathrm{BF}_{10}$ was interpreted in accordance with Lee and Wagenmaker's classification categories [45]. We calculated variance inflation factor (VIF)

\footnotetext{
${ }^{2}$ The experimenter learned from the participants that the name of this character is actually 'Steve'.

${ }^{3}$ https://www.psytoolkit.org/experiment-library/
} 
to confirm that the independent variables did not exhibit a problematic amount of collinearity ${ }^{4}$ (VIF for all variables $<1.7)$.

\section{RESULTS}

\subsection{Audience design and age}

Non-transformed AD score by age group was: $11-12$ years: $M=0.52$, std. error $=0.06,15-16$ years: $M$ $=0.78$, std. error $=0.06$ (Figure 2). Compared to their younger peers, 15-16-year-olds described referents they assumed to be unknown to addressees more often (in $93 \%$ vs. $83 \%$ of unknown condition trials), and described referents they assumed to be known to their addressees less often (in $16 \%$ vs. $30 \%$ of known condition trials). The frequentist GLM with AD as dependent variable and AGE GROUP, KAS, PE and $\mathrm{NE}$ as independent variables (Model 1a) revealed a significant effect of AGE GROUP on AD performance: $T(53)=3.7, p<.001$ ). The corresponding Bayesian GLM (Model 1b) found strong evidence for H1 with respect to AGE GROUP $\left(\mathrm{BF}_{10}=20\right)$. A similar effect of AGE GROUP was observed with the non-transformed data: Model 1a: $T(53)=3.4, p<.01$ ), Model 1b: $\mathrm{BF}_{10}=16$ (strong evidence for $\mathrm{H} 1$ ).

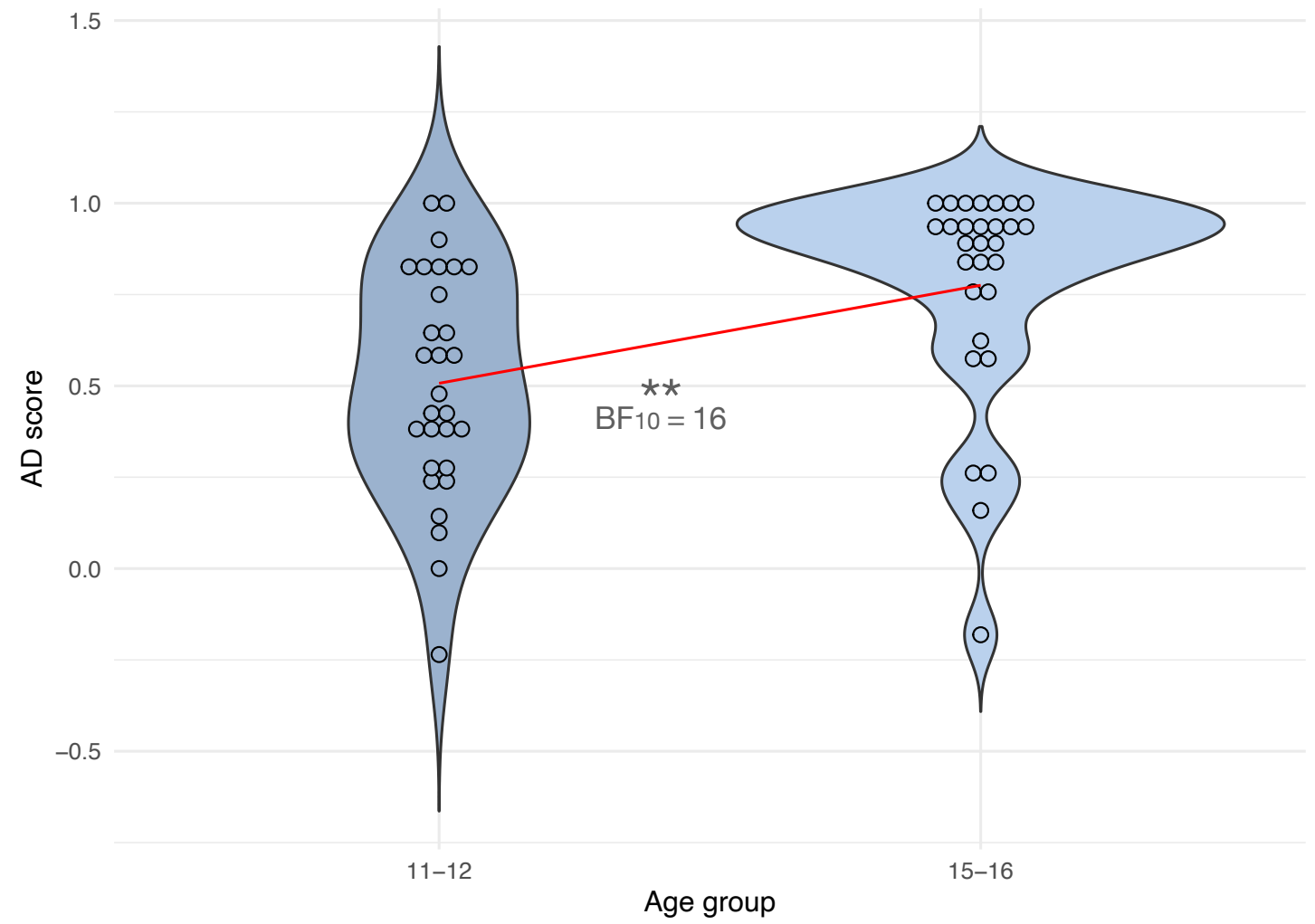

Figure 2. Non-transformed individual AD score by age group. The red line indicates the development of mean ability to adapt utterances to the assumed frame of reference of the addressee.

\subsection{Audience design and matching theory of mind information}

Model 1a found no effect of KAS on AD performance: $T(53)=0.7, p=.5$ (Figure 3). No evidence for an effect of KAS on AD score was found in Model $1 \mathrm{~b}(\mathrm{BF} 10=1 / 15$, strong evidence against H1). There was no effect of AGE GROUP on KAS in Model 2 (11-12 years: $\mathrm{M}=.94$, std. error $=0.01$, 15-16 years: $\mathrm{M}=$ .96 , std. error $<0.01, F(1,56)=1.3, p=.26)$.

\subsection{Audience design and cognitive control functions}

No effect of PE or NE on AD performance was found in Model 1a (PE: $T(53)=1.2, p=.25 ;$ NE: $T(53)$ $=0.4, p=.7)$ ) (Figure 3). No evidence for a effect of PE nor NE was found on AD performance in

\footnotetext{
${ }^{4}$ VIF values greater than 5 are assumed to indicate a problematic amount of collinearity [46].
} 

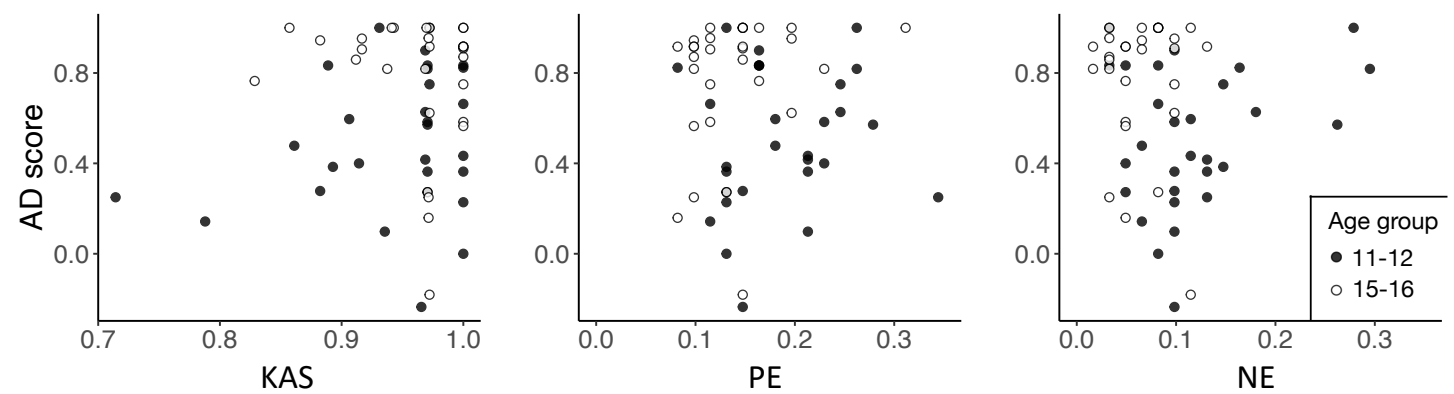

Figure 3. Relation between AD performance (non-transformed scores) and KAS (knowledge attribution score, indicating ToM; leftmost panel), PE (perseverative errors, indicating ability to flexibly switch matching rule after set-shift in the WCST; middle panel), and NE (non-perseverative errors, indicating ability to maintain a correct matching behavior during a set in the WCST; rightmost panel). Data points represent individual performance and are color-coded by age group.

Model $1 \mathrm{~b}\left(\mathrm{PE}: \mathrm{BF}_{10}=1 / 8\right.$, moderate evidence against $\mathrm{H} 1 ; \mathrm{NE}: \mathrm{BF}_{10}=1 / 14$, strong evidence against $\left.\mathrm{H} 1\right)$. There was a significant effect of AGE GROUP on NE (Model 3) and PE (Model 4) respectively (PE for 11-12-year-olds: $\mathrm{M}=0.18$, std. error $=0.01$, and 15-16-year-olds: $\mathrm{M}=0.14$, std. error $=0.009, F(1,56)=$ 9.55, $p<.01$; $\mathrm{NE}$ for 11-12-year-olds: $\mathrm{M}=0.11$, std. error $=0.012$, and 15-16-year-olds: $\mathrm{M}=0.07$, std. error $=0.005, F(1,56)=13.89, p<.001)$.

\section{DISCUSSION}

Using referential production as a test bed, we have shown a significant development in audience design from the time period where children enter adolescence until mid adolescence (AD). Our results show that the younger age group (11-12) do not utilize relevant ToM information during online production to the same extent as the older age group (15-16), see Figure 2. We provide evidence that this development cannot be explained by measures of their individual ToM information (as there was no such relationship, see Figure 3). Nor was there a development of ToM information across age groups (Bayes factor showed strong evidence against such an effect). In other words, the relevant ToM information was already present and ready to be accessed in early adolescence, but only when the adolescents were specifically prompted for this information. Furthermore, an expected age-related development of CCF was observed across groups but there was no link between audience design (AD) and CCF, see Figure 3. We thus provide evidence suggesting that 11-12- and 15-16-year-olds' ability to engage in AD with respect to adapting utterances to the inferred world knowledge of hearers cannot be explained by measures of their CCF.

These findings support accounts from referential comprehension, suggesting that adolescence is a period of significant growth with respect to the ability to utilize ToM during online communication [15]. While 7-year-old children fail to flexibly adapt their utterances to the assumed world knowledge of interlocutors [3], adolescence appears to be a sensitive period in this respect. This may be ascribed to the functional reorganizations and structural changes of brain regions important to perspective taking during online communication that take place in adolescence $[21,22,47]$. The ability to engage in world knowledge-based $\mathrm{AD}$ does not seem to reflect a development in ability to correctly infer the knowledge state of an addressee (since there was no observed difference in the KAS score across age groups). This supports a processing model distinguishing between ToM information as such and the usage of ToM during online communication [23].

Our AD task was designed to tap ToM-processing. Few have conducted such tasks on adolescents, potentially due to risks of producing ceiling effects [21]. The task could be considered as a suitable measure of adolescents' online ToM usage in language production, since it generated notable variance between age groups. This is particularly important, considering that the ability to use ToM information in communicative contexts has been shown to correlate with adolescents' self-reported peer relations [48], which in turn play a deciding role in their psychological well-being [49]. Constructing paradigms that sufficiently measure adolescents' pragmatic production has the potential to further the understanding of late pragmatic development and its relation to other aspects of cognition, such as ToM and CCF, but also "core" language ability (see section 4.1) and attention). Research on AD in referential communication 
typically uses visual perspective as its paradigm mental state $[4,15,50]$, and world knowledge of interlocutors has gone systematically overlooked. Our world knowledge-based AD task contributes to the debate regarding whether frequently utilized referential communication paradigms tap general cognitive processes (such as executive operations or selective attention) rather than ToM processing $[6,20]$. This debate is important since such tasks, i.e., the "director task" (DT) [18] or the "multiple features task" [51], have been utilized to argue that cognitive control functions play a crucial role in online communicative ToM usage $[23,52]$. Firstly, consider that unlike the DT, our task was designed so that successful performance required participants to consider the world knowledge of interlocutors by taking into account listener-specific features such as age and gender. Secondly, unlike the "multiple features task", our task did not necessitate the retainment of multiple features of competing objects (e.g. color, size and/or number) in memory, which in itself may put a high demand on cognitive processing [51].

Why do not children entering adolescence routinely use information about the world knowledge of the interlocutor during online production, even when this information is accessible? From a neurobiological perspective, the functional reorganizations and structural changes of brain regions important to perspective taking during online communication that take place in adolescence [21, 22, 47], may be ascribed as a causal explanation. Co-development of these neural changes and functional changes are also likely. One possible functional explanation of this intriguing pattern is that adult interlocutors have systematically been covering up for this lack of perspective taking, for instance, by making assumptions or requesting clarification (e.g., "Is Minecraft the game that mom brought you for Christmas?"). Upon entering adolescence, social motivation is increased to succeed in the peer group, and cultural references proliferate into adolescent subcultures, increasing the need to systematically take this aspect of ToM into account. Conceivably, as an individual approaches adulthood, departures from being a cooperative interlocutor may more often result in conversational breakdown, giving rise to more frequent learning opportunities.

Another perspective on this development, proposed by Grigoroglou \& Papafragou [53], is that some listener-specific adjustments, (also termed particular-listener adjustments [54]), may be computationally simpler (e.g., adjustments based on knowledge shared by joint experience, as in classical false belief tasks [12]) and thus emerge earlier than costlier adaptations. There is evidence supporting this view; while consistent success in false belief tasks is achieved near universally by typically developed 5- to 6-year-olds [55-57], 5-6-year old's do not consistently provide sufficiently informative expressions in order to disambiguate between competing objects that are visually accessible to the addressee (in $75 \%$ of trials) [4]. Building on this perspective, world knowledge-based adjustments may develop even later: 7-year-olds adapted their utterances when the referent was unknown to the addressee in $32 \%$ of trials [3], and our findings suggest that this ability continues to successively develop in adolescence. Ultimately, variation in pragmatic processing developmentally could be linked to adult individual differences in AD, which are large [6].

Which are the underlying factors for late and successive development of AD? Listener-specific adjustments in production have been described as possibly relying on 3 critical and interrelated components: (1) experience with social interaction, (2) ability to reason about the knowledge states to others (reflected by our KAS score), and (3) CCF (reflected by our WCST scores) [23, 28]. Our findings suggest that the development of world-knowledge based $\mathrm{AD}$ cannot be reduced simply to the maturation of knowledge state-reasoning or CCF. Measuring and testing for amount of social experience (which can be assumed to increase with age) is of course difficult, but we can turn to what is acquired from experience of social interaction. For example, it has previously been suggested that pragmatic competence should be regarded as more than a composition of other more domain-general skills, but a specific capability with its own characterization in terms of developmental trajectory and neurophysiological correlates $[6,58]$. This would explain the mixed findings with respect to the relation between $\mathrm{AD}$ and more domain-general cognitive skills [13, 28, 48, 52, 59].

\subsection{Limitations}

Referential production tasks conducted with passive confederates (as in the current study and [3,6, 60]) have been criticized since interactive contexts increase the informativeness of both child and adult speakers' referential expressions [61]. Motivation to adjust referential expressions to hearers may have been increased with the current paradigm if hearers would have been active conversational partners, potentially even known to the participant. We did however conduct the experiment with speaker motivation in mind and, apart from giving a detailed account of the two addressees so that speaker's would have 
a clear representation of them, we presented the task as a challenge in which success depended on whether the addressee would be able to pick the correct referent when listening to the speaker's directions. Furthermore, it is possible the observed variance between the age groups does reflect a difference in level of automation in the behavior of adapting utterances to the inferred knowledge of others.

Demands on formal language was controlled for in the current AD-task as each referent description only needed to consist of a noun phrase with a maximum of one modifier (an adjectival or a prepositional phrase). Individual language ability was however not included as a variable in our models. Many developmental studies investigating formal language (i.e., vocabulary or morphosyntax) abilities as predictors of pragmatic ability find a medium to strong correlation, although evidence from referential communication is mixed (for a review, see [28]). Results from 7-year-olds' performance on a world knowledge-based AD task did not correlate significantly with measures of receptive vocabulary (collected 3 years earlier) or with measures of syntactic production [62]. Problem-solving involved in WCST has been linked to covert language processes [63], but the task does not rely on formal language skills as directly as other measures of CCF (consider for example the Stroop tasks, which generally involve word reading [64]). In summary, the influence of formal language abilities on the current results are judged to be minimal.

Although the WCST is regarded as an ecologically valid measure of CCF [65], there are some uncertainties regarding which specific CCF subfunctions the WCST taps. Individual WCST scores do not reflect individual CCF subfunctions [66], and the scores used in this study (proportion perseverative errors and proportion non-perseverative errors) have been shown to mainly correlate with working memory and cognitive flexibility in adolescence $[35,36]$. In consequence, it is possible that some subfunction that was not reflected in the WCST scores, such as inhibition (although correlating with WCST non-perseverative errors in 7-year-olds [36]), played a role in enabling successful AD performance.

\subsection{Conclusions}

We present evidence that the ability to engage in world knowledge-based AD continues to develop between late childhood (11-12) and mid adolescence (15-16). Children entering adolescence used ToM information in production to a notably low extent, although the relevant ToM information was accessed upon subsequent prompting to the same extent as in the older group. This supports a processing model distinguishing between ToM information as such and the usage of ToM during online communication, where the latter develops later. When it comes to using world knowledge as a ToM information type, our data suggest a developmental timeline spanning adolescence, possibly resulting in adult individual differences. Furthermore, we provide empirical support for the notion that an age-related increase in CCF does not explain age-related development of world knowledge-based audience design during online production. In summary, our results speak in favor of continued adolescent development in pragmatic processing, over and above development of ToM and cognitive control functions.

\section{REFERENCES}

1. Piaget J. Judgment and reasoning in the child. London: Routledge \& Kegan Paul PLC, 1928/2002

2. Clark HH and Murphy GL. Audience Design in Meaning and Reference. Advances in Psychology. Ed. by Le Ny JF and Kintsch W. Vol. 9. Language and Comprehension. North-Holland, 1982 :287-99. DOI: $10.1016 /$ S0166-4115(09) 60059-5

3. Pagmar D, Arvidsson C, and Uddén J. Testing for Audience Design: a short report. 2021 Sep. DoI: 10.31234/osf.io/3d7ja. Available from: psyarxiv.com/3d7ja

4. Nadig A and Sedivy JC. Evidence of Perspective-Taking Constraints in Children's On-Line Reference Resolution. Psychological Science 2002; 13. Publisher: SAGE Publications Inc:329-36. DOI: $10.1111 / j .0956-7976.2002 .00460 . x$

5. Grice HP. Logic and conversation. Speech acts. Ed. by Cole P and Morgan JL. Brill, 1975 :41-58

6. Bendtz K, Eriksson S, Schneider J, Borg J, Basnakova J, and Uddén J. Pragmatics as more than core language plus theory of mind: an individual differences study using fMRI. Neurobiology of language 2022; Accepted

7. Clark HH. Using language. Cambridge university press, 1996 
8. Horton WS and Keysar B. When do speakers take into account common ground? Cognition 1996; 59:91-117

9. Astington JW, Harris PL, and Olson DR. Developing theories of mind. Cambridge University Press, 1988

10. Premack D and Woodruff G. Does the chimpanzee have a theory of mind? Behavioral and brain sciences $1978 ; 1: 515-26$

11. Wellman HM. The child's theory of mind. The MIT Press, 1992

12. Perner J and Leekam SR. Belief and quantity: Three-year olds' adaptation to listener's knowledge. Journal of Child Language 1986; 13:305-15

13. Nilsen ES and Graham SA. The relations between children's communicative perspective-taking and executive functioning. Cognitive Psychology 2009; 58:220-49. DOI: 10.1016/j.cogpsych. 2008.07 .002

14. Branigan HP, Bell J, and McLean JF. Do you know what I know? The impact of participant role in children's referential communication. Frontiers in psychology 2016; 7:213

15. Dumontheil I, Apperly IA, and Blakemore SJ. Online usage of theory of mind continues to develop in late adolescence. Developmental Science 2010; 13:331-8

16. Gann $\mathrm{T}$ and Barr DJ. Speaking from experience: audience design as expert performance. Language, Cognition and Neuroscience 2014; 29:744-60. DOI: $10.1080 / 01690965.2011 .641388$

17. Loy JE, Bloomfield SJ, and Smith K. Effects of Priming and Audience Design on the Explicitness of Referring Expressions: Evidence From a Confederate Priming Paradigm. Discourse Processes 2020; 57:808-21

18. Keysar B, Barr DJ, Balin JA, and Brauner JS. Taking perspective in conversation: The role of mutual knowledge in comprehension. Psychological Science 2000; 11:32-8

19. Fukumura K. Development of audience design in children with and without ASD. Developmental Psychology 2016; 52:71-87. DOI: $10.1037 /$ dev0000064

20. Rubio-Fernández P. The director task: A test of Theory-of-Mind use or selective attention? Psychonomic Bulletin \& Review 2017; 24:1121-8

21. Blakemore SJ. The social brain in adolescence. Nature Reviews Neuroscience 2008; 9:267-77

22. Vanlangendonck F, Willems RM, and Hagoort P. Taking common ground into account: Specifying the role of the mentalizing network in communicative language production. PloS One 2018; 13:e0202943

23. Nilsen ES and Fecica AM. A model of communicative perspective-taking for typical and atypical populations of children. Developmental Review 2011; 31:55-78

24. Miller EK. The prefontral cortex and cognitive control. Nature reviews neuroscience 2000; 1:59-65

25. Deák GO. The Development of Cognitive Flexibility and Language Abilities. Advances in Child Development and Behavior. Vol. 31. JAI, 2004 :271-327. DOI: 10.1016/S0065-2407 (03) 31007-9

26. Diamond A. The Early Development of Executive Functions. Lifespan Cognition: Mechanisms of Change $2002: 70-95$

27. Leon-Carrion J, Garcia-Orza J, and Pérez-Santamarıa FJ. Development of the inhibitory component of the executive functions in children and adolescents. International Journal of Neuroscience 2004; 114:1291-311

28. Matthews D, Biney H, and Abbot-Smith K. Individual Differences in Children's Pragmatic Ability: A Review of Associations with Formal Language, Social Cognition, and Executive Functions. Language Learning and Development 2018; 14:186-223. DOI: $10.1080 / 15475441.2018$. 1455584

29. Bacso $\mathrm{S}$ and Nilsen ES. What's that you're saying? Children with better executive functioning produce and repair communication more effectively. Journal of Cognition and Development 2017; 18:441-64 
30. Peirce JW. PsychoPy—psychophysics software in Python. Journal of Neuroscience Methods 2007; $162: 8-13$

31. Cummings L. Cognitive aspects of pragmatic disorders. Research in clinical pragmatics. Springer, $2017: 587-616$

32. Grant DA and Berg EA. Wisconsin Card Sorting Test (WCST). PAR., 1993

33. Barceló F and Knight RT. Both random and perseverative errors underlie WCST deficits in prefrontal patients. Neuropsychologia 2002; 40:349-56

34. Anokhin AP, Heath AC, and Ralano A. Genetic influences on frontal brain function: WCST performance in twins. Neuroreport 2003; 14:1975-8

35. Huizinga M, Dolan CV, and Van der Molen MW. Age-related change in executive function: Developmental trends and a latent variable analysis. Neuropsychologia 2006; 44:2017-36

36. Huizinga $\mathrm{M}$ and Van der Molen MW. Age-group differences in set-switching and set-maintenance on the Wisconsin Card Sorting Task. Developmental Neuropsychology 2007; 31:193-215

37. Landry $\mathrm{O}$ and Mitchell $\mathrm{P}$. An examination of perseverative errors and cognitive flexibility in autism. Plos one 2021; 16:e223160

38. Stoet G. PsyToolkit: A software package for programming psychological experiments using Linux. Behavior Research Methods 2010; 42:1096-104

39. Stoet G. PsyToolkit: A novel web-based method for running online questionnaires and reaction-time experiments. Teaching of Psychology 2017; 44:24-31

40. Bishara AJ and Hittner JB. Testing the Significance of a correlation with nonnormal data: Comparison of Pearson, Spearman, transformation, and resampling approaches. Psychological Methods 2012; 17:399-417

41. Arvidsson C, Pagmar D, and Uddén J. Adolescent development of world knowledge-based audience design. Dataset. 2022. DOI: 10.17605 / OSF . IO/4Y8BS. Available from: https: / / osf . io/4y8bs/

42. R Core Team. R: A Language and Environment for Statistical Computing. R Foundation for Statistical Computing. Vienna, Austria, 2021

43. Gabry J and Goodrich B. rstanarm: Bayesian applied regression modeling via Stan. R package version 2.10. 0. 2016

44. Makowski D, Ben-Shachar MS, and Lüdecke D. bayestestR: Describing effects and their uncertainty, existence and significance within the Bayesian framework. Journal of Open Source Software 2019; $4: 1541$

45. Lee MD and Wagenmakers EJ. Bayesian cognitive modeling: A practical course. Cambridge university press, 2014

46. James G, Witten D, Hastie T, and Tibshirani R. An introduction to statistical learning. Vol. 112. Springer, 2013

47. Giedd JN, Blumenthal J, Jeffries NO, Castellanos FX, Liu H, Zijdenbos A, Paus T, Evans AC, and Rapoport JL. Brain development during childhood and adolescence: a longitudinal MRI study. Nature Neuroscience $1999 ; 2: 861-3$

48. Nilsen ES and Bacso S. Cognitive and behavioural predictors of adolescents' communicative perspective-taking and social relationships. Journal of Adolescence 2017; 56:52-63. DOI: 10 . 1016/j.adolescence.2017.01.004

49. Corsano P, Majorano M, and Champretavy L. Psychological well-being in adolescence: the contribution of interpersonal relations and experience of being alone. Adolescence 2006; 41

50. Epley N, Morewedge CK, and Keysar B. Perspective taking in children and adults: Equivalent egocentrism but differential correction. Journal of Experimental Social Psychology 2004; 40:760-8. DOI: $10.1016 / j . j e s p .2004 .02 .002$ 
51. Dahlgren S and Sandberg A. Referential communication in children with autism spectrum disorder. Autism : the International Journal of Research and practice 2008; 12:335-48. DOI: 10.1177 / 1362361308091648

52. Nilsen ES, Varghese A, Xu Z, and Fecica A. Children with stronger executive functioning and fewer ADHD traits produce more effective referential statements. Cognitive Development 2015 ; 36:68-82. DOI: $10.1016 / j . c o g d e v .2015 .09 .001$

53. Grigoroglou M and Papafragou A. Children's (and Adults') production adjustments to generic and particular listener needs. Cognitive science 2019; 43:e12790

54. Brown PM and Dell GS. Adapting production to comprehension: The explicit mention of instruments. Cognitive psychology 1987; 19:441-72

55. Peterson CC and Wellman HM. Longitudinal theory of mind (ToM) development from preschool to adolescence with and without ToM delay. Child Development 2019; 90:1917-34

56. Callaghan T, Rochat P, Lillard A, Claux ML, Odden H, Itakura S, Tapanya S, and Singh S. Synchrony in the onset of mental-state reasoning: Evidence from five cultures. Psychological Science 2005; 16:378-84

57. Wellman HM, Cross D, and Watson J. Meta-analysis of theory-of-mind development: The truth about false belief. Child development 2001; 72:655-84

58. Domaneschi F and Bambini V. Pragmatic competence. 2020

59. Saryazdi R and Chambers CG. Real-time communicative perspective taking in younger and older adults. Journal of Experimental Psychology: Learning, Memory, and Cognition 2020. Place: Washington, US Publisher: American Psychological Association (US). DOI: http://dx . doi . org/10.1037/xlm0000890

60. Davies C, Andrés-Roqueta C, and Norbury CF. Referring expressions and structural language abilities in children with specific language impairment: A pragmatic tolerance account. Journal of Experimental Child Psychology 2016; 144:98-113. DoI: 10.1016/j.jecp.2015.11.011

61. Grigoroglou M and Papafragou A. Interactive contexts increase informativeness in children's referential communication. Developmental Psychology 2019; 55:951-66. DOI: 10.1037/dev0000693

62. Pagmar D, Arvidsson C, Gerholm Nilsson T, and Uddén J. Conversation skill at school start and connections to executive functions and conversational implicatures. 2021; Manuscript in preparation

63. Baldo JV, Dronkers NF, Wilkins D, Ludy C, Raskin P, and Kim J. Is problem solving dependent on language? Brain and Language 2005; 92:240-50

64. Williams JMG, Mathews A, and MacLeod C. The emotional Stroop task and psychopathology. Psychological Bulletin 1996; 120:3

65. Kibby MY, Schmitter-Edgecombe M, and Long CJ. Ecological validity of neuropsychological tests: focus on the California Verbal Learning Test and the Wisconsin Card Sorting Test. Archives of Clinical Neuropsychology 1998; 13:523-34

66. Romine CB, Lee D, Wolfe ME, Homack S, George C, and Riccio CA. Wisconsin Card Sorting Test with children: a meta-analytic study of sensitivity and specificity. Archives of Clinical Neuropsychology 2004; 19:1027-41 